\title{
The memory-impairing effects of septal GABA receptor activation involve GABAergic septo-hippocampal projection neurons
}

\author{
Desiree L. Krebs-Kraft, ${ }^{1,2,3,4}$ Marina G. Wheeler, ${ }^{1,2}$ and Marise B. Parent ${ }^{1,2,5}$ \\ ${ }^{1}$ Department of Psychology, Georgia State University, Atlanta, Georgia 30303, USA; ${ }^{2}$ Center for Behavioral Neuroscience, \\ Georgia State University, Atlanta, Georgia 30303, USA; ${ }^{3}$ Department of Physiology, University of Maryland, Baltimore, \\ Maryland 21201, USA; ${ }^{4}$ Program in Neuroscience, University of Maryland, Baltimore, Maryland, 21201, USA
}

\begin{abstract}
Septal infusions of the $\gamma$-aminobutyric acid $(G A B A)_{A}$ agonist muscimol impair memory, and the effect likely involves the hippocampus. $\mathrm{GABA}_{\mathrm{A}}$ receptors are present on the perikarya of cholinergic and GABAergic septo-hippocampal $(\mathrm{SH})$ projections. The current experiments determined whether GABAergic SH projections are involved in the memory-impairing effects of septal $G_{A B A}$ receptor activation. Experiment 1 tested whether combining septal co-infusions of subeffective doses of muscimol with scopolamine, a drug that selectively influences GABA SH projections, would produce memory deficits. Experiment 2 tested whether hippocampal infusions of a $\mathrm{GABA}_{\mathrm{A}}$ receptor antagonist would block the effects of septal muscimol infusions. Fifteen minutes prior to assessing spontaneous alternation (SA) or training in a multiple trial inhibitory avoidance (CMIA) task, male Sprague-Dawley rats were given septal infusions of vehicle, muscimol, scopolamine, or co-infusions of muscimol with scopolamine, or septal infusions of vehicle or muscimol combined with hippocampal infusions of vehicle or bicuculline. Septal co-infusions of muscimol with scopolamine significantly impaired SA and CMIA. Hippocampal bicuculline infusions blocked deficits produced by septal muscimol infusions in SA and attenuated deficits produced in CMIA. Combined, these findings suggest that GABAergic SH projections are involved in the memory-impairing effects of septal GABA receptor activation.
\end{abstract}

Activation of $\gamma$-aminobutyric acid $(\mathrm{GABA})_{\mathrm{A}}$ receptors in the medial septum (MS) impairs memory in a variety of learning and memory tasks (Chrobak et al. 1989; Brioni et al. 1990; Durkin 1992; Nagahara and McGaugh 1992; Pang and Nocera 1999). These impairing effects of $\mathrm{MS} \mathrm{GABA}_{\mathrm{A}}$ receptor activation likely involve the hippocampus. For instance, MS infusions of the $\mathrm{GABA}_{\mathrm{A}}$ agonist muscimol impair hippocampal theta (Allen and Crawford 1984; Smythe et al. 1992; Wan et al. 1995), which is a rhythmic oscillation important for memory (O'Keefe 1993; Vertes and Kocsis 1997; Hasselmo et al. 2002; Hasselmo 2005). Also, the memory-impairing effects of MS infusions of muscimol are reversed by hippocampal infusions of glucose, pyruvate, or the acetylcholinesterase (AChE) inhibitor physostigmine (Parent et al. 1997; Degroot and Parent 2000, 2001; Krebs and Parent 2005a,b).

The MS is connected to the hippocampus via the fimbriafornix, which is composed primarily of cholinergic and GABAergic projection neurons (Lewis et al. 1967; Kohler et al. 1984; Rye et al. 1984; Fig. 1B). Although a glutamatergic projection was recently demonstrated, very little is known about the connectivity or receptor makeup of this septo-hippocampal (SH) projection (Sotty et al. 2003; Colom et al. 2005). GABAergic SH projection neurons synapse onto hippocampal GABA interneurons (Freund and Antal 1988), which in turn synapse onto glutamatergic pyramidal cells (Toth et al. 1997). GABAergic SH afferents, therefore, produce a net disinhibition of hippocampal pyramidal cells (Bilkey and Goddard 1985; Krnjevic et al. 1988). Cholinergic SH projection neurons terminate broadly in the hippocampus, synapsing onto pyramidal cells, dentate granule cells, and inhibitory interneurons (Frotscher and Leranth 1985). Activation of cholinergic $\mathrm{SH}$ projection neurons excites pyramidal cells, which would

${ }^{5}$ Corresponding author.

E-mail mbparent@gsu.edu; fax (404) 651-3929.

Article is online at http://www.learnmem.org/cgi/doi/10.1101//m.809407. be expected to have positive effects on memory (Teyler 1987; Izquierdo and Medina 1993; Wu et al. 2002; Fig. 1B).

$\mathrm{GABA}_{\mathrm{A}}$ receptors are present on the cell bodies of both GABAergic and cholinergic SH neurons (Gao et al. 1995). The memory-impairing effects of $\mathrm{MS} \mathrm{GABA}_{\mathrm{A}}$ receptor activation could therefore involve either or both $\mathrm{SH}$ projection neurons. Extensive evidence indicates that cholinergic SH projection neurons are involved in the memory-impairing effects of muscimol. For example, MS infusions of muscimol, at doses that impair memory, decrease hippocampal extracellular acetylcholine (ACh) levels (Durkin 1992; Gorman et al. 1994; Degroot et al. 2003). Furthermore, only those doses of muscimol that decrease hippocampal ACh impair memory (Brioni et al. 1990). Also, manipulations that increase hippocampal ACh levels reverse the memory deficits produced by MS GABA receptor activation (Parent et al. 1997; Degroot and Parent 2000, 2001; Krebs and Parent 2005a). Furthermore, MS GABA receptor activation increases the dose of ACh receptor agonists needed in the hippocampus to reverse memory deficits (Farr et al. 1999).

More recent evidence suggests that GABAergic SH projection neurons may also be involved in memory. Ibotenic and kainic acid lesions, which primarily affect GABAergic SH neurons, impair learning (Cahill and Baxter 2001; Dwyer et al. 2007). Selective lesions of cholinergic SH neurons do not prevent the memory-impairing effects of muscimol (Pang and Nocera 1999), further suggesting that GABAergic $\mathrm{SH}$ projection neurons are involved in memory. Moreover, electrophysiological studies show that MS administration of ACh agonists, which can have memory-enhancing actions within a certain range (Givens and Olton 1990, 1995), selectively excites GABAergic SH projection neurons, but not cholinergic SH projection neurons (Wu et al. 2000). Similarly, the muscarinic ACh receptor antagonist scopolamine, a drug that impairs memory when infused into the MS (Pang and Nocera 1999; Elvander et al. 2004), also influ- 
A

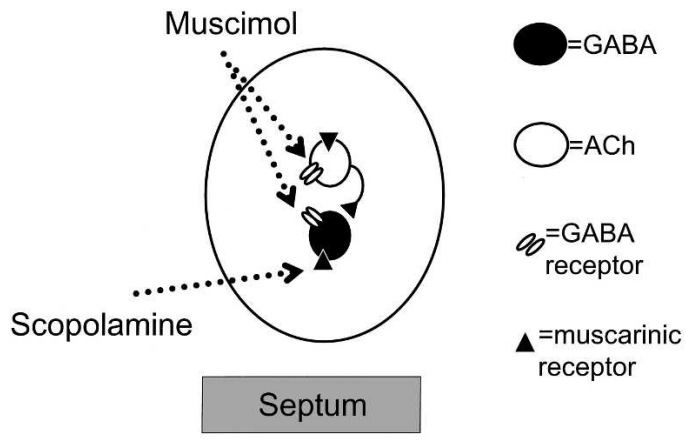

B

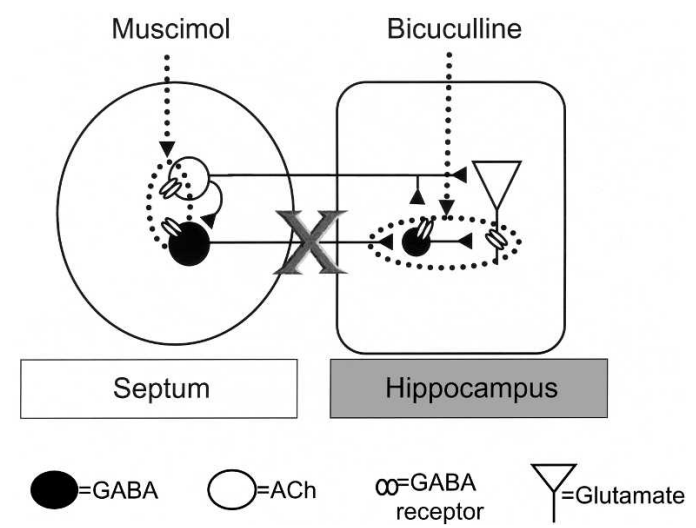

Figure 1. (A) Schematic illustration of the MS. Muscarinic receptors are present on the cell bodies of both ACh and GABA projection neurons (Van der Zee and Luiten 1994). MS administration of scopolamine inhibits only the activity of GABAergic SH projection neurons (Alreja et al. 2000). If MS co-infusions of muscimol with scopolamine impair memory, then these results would suggest that the impairing affects of muscimol also involve GABAergic SH projection neurons. (B) Schematic illustration of the septo-hippocampal system. Muscimol infused into the MS binds to GABA receptors present on both cholinergic and GABAergic SH projection neurons (Gao et al. 1995) and inhibits the activity of both projection neurons (M. Alreja, pers. comm.). Importantly, in this concurrent infusion paradigm, the hippocampal infusions of the GABA antagonist are blocking the contributions of the GABAergic SH projection neurons. We assume that the hippocampal bicuculline infusions are not preventing the effects of the ACh collaterals onto the GABAergic SH projection neurons, because presumably these ACh collaterals are already inhibited by the MS GABA receptor activation. As a result, if hippocampal infusions of bicuculline reverse the deficits produced by MS infusions of muscimol, then this will suggest that GABAergic SH projection neurons are involved in the impairing effects of muscimol.

ences GABAergic SH projection neurons selectively (Alreja et al. 2000).

Although the evidence reviewed above suggests that GABAergic $\mathrm{SH}$ projection neurons are involved in memory, there is no direct behavioral evidence to that effect. Consequently, the goal of the present research was to determine whether GABAergic $\mathrm{SH}$ projection neurons are involved in the memory-impairing effects of $\mathrm{MS} \mathrm{GABA}_{\mathrm{A}}$ receptor activation. Experiment 1 determined whether combining septal infusions of subeffective doses of the GABA agonist muscimol with the muscarinic antagonist scopolamine, a drug that selectively influences GABAergic SH projection neurons (Alreja et al. 2000), would summate to produce memory deficits (Fig. 1A). Such a finding would suggest that muscimol and scopolamine are acting on a common mechanism (i.e., GABAergic SH projection neurons) to impair memory (Seeley and Moran 2002). Experiment 2 determined whether hippocampal infusions of the $\mathrm{GABA}_{\mathrm{A}}$ receptor antagonist bicuculline would prevent the memory-impairing effects of MS infusions of muscimol. MS infusions of muscimol should inhibit SH GABA projection neurons, thus disinhibiting GABA interneurons (Fig. 1B). This disinhibition of GABA interneurons would, in turn, increase inhibition of pyramidal cells. Hippocampal infusions of bicuculline would be expected to block the inhibitory effects of the interneurons onto the pyramidal cells. Thus, combining hippocampal infusions of bicuculline with MS infusions of muscimol should selectively prevent the influence of GABAergic $\mathrm{SH}$ projection neurons on hippocampal pyramidal cells via GABA interneurons (Fig. 1B). For both experiments, rats participated in two hippocampal-dependent memory tasks that vary in motivational, temporal, and cognitive demands. This would allow us to assess whether the manipulations were affecting memory rather than some process that influences performance on the memory task.

\section{Results}

\section{Experiment 1}

The locations of the MS infusions are shown in Figure 2. Drug infusions into the MS significantly affected SA performance
$\left(F_{(3,62)}=4.26 ; P<0.01\right.$; Fig. 3A). MS infusions of muscimol or scopolamine alone did not impair SA performance. Specifically, the percent alternation scores of rats given MS infusions of muscimol or scopolamine alone were not significantly different from those of rats given infusions of vehicle $(P>0.05)$. Interestingly, the findings showed that MS co-infusions of muscimol with scopolamine significantly impaired SA performance. Specifically, the percent alternation scores of rats given muscimol and scopolamine in the same solution were significantly lower than those of rats given MS infusions of vehicle $(P<0.05)$. However, the percent alternation scores of rats given muscimol and scopolamine in the same solution were not significantly different from those of rats given MS infusions of scopolamine or muscimol alone (Ps > 0.05). Drug infusions into the MS significantly affected the number of arms that the rat entered in the maze $\left(\mathrm{F}_{(3,62)}=2.95 ; P<0.05\right.$; Fig. $\left.3 \mathrm{~B}\right)$. The data showed that the MS infusions of scopolamine alone decreased the number of arms the rats entered in the maze. Specifically, the rats given MS infusions of scopolamine alone entered fewer arms than did rats given vehicle infusions $(P<0.05)$.

MS drug infusions did not significantly affect trials to criterion during CMIA training $\left(\chi_{(3,57)}^{2}=2.04 ; P>0.05\right.$; Fig. 4A). Figure $4 \mathrm{~B}$ illustrates that the pre-training drug infusions into the MS significantly affected subsequent CMIA retention performance tested $48 \mathrm{~h}$ later $\left(\chi^{2}{ }_{(3,57)}=7.92 ; P<0.05\right)$. MS infusions of muscimol or scopolamine alone did not significantly impair CMIA retention. Specifically, the retention latency scores of rats that were given MS infusions of muscimol $\left(U_{(1,30)}=98.5\right.$; $P>0.05)$ or scopolamine $\left(\mathrm{U}_{(1,33)}=97.5 ; P>0.05\right)$ alone were not different from those of rats that were given MS infusions of vehicle. Importantly, MS co-infusions of muscimol with scopolamine impaired avoidance retention. The retention latencies of rats that were given MS co-infusions of muscimol with scopolamine were significantly lower than those of rats that were given MS infusions of vehicle $\left(\mathrm{U}_{(1,30)}=51 ; P<0.01\right)$. However, the retention latencies of rats that were given MS co-infusions of muscimol with scopolamine were not significantly lower than those of rats that were given MS infusions of scopolamine $\left(\mathrm{U}_{(1,27)}=86\right.$; $P>0.05)$ or muscimol alone $\left(\mathrm{U}_{(1,24)}=51.5 ; P>0.05\right)$. 


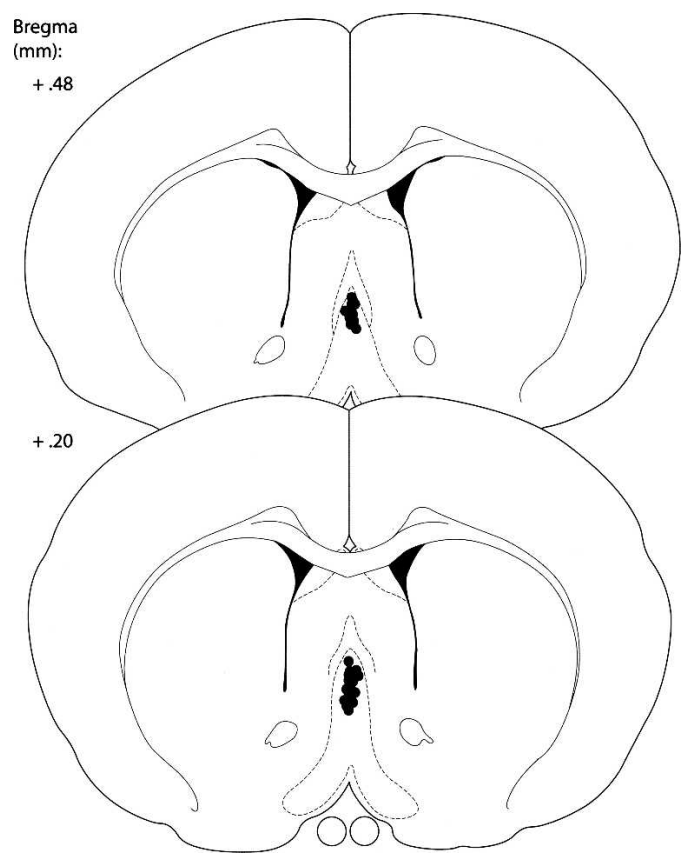

Figure 2. Schematic illustration of coronal sections of the rat brain showing the approximate location of MS infusion sites in Experiment 1. Atlas plates were adapted from Paxinos and Watson (1998), with permission from Elsevier (C1998.

\section{Experiment 2}

Figure 5 illustrates the approximate locations of MS and hippocampal infusions. Figure $6 \mathrm{~A}$ illustrates that drug infusions into the MS $\left(F_{(1,42)}=5.611 ; P<0.05\right)$ and the hippocampus $\left(F_{(1,42)}=9.900 ; P<0.05\right)$ significantly affected percent alternation scores, and the effects of drug infusions into both regions interacted significantly $\left(F_{(1,42)}=4.542 ; P<0.05\right)$. Hippocampal infusions of bicuculline alone did not affect percent alternation scores. Compared with the percent alternation scores of rats that were given infusions of vehicle in both brain regions $(\mathrm{V}-\mathrm{V})$, the percent alternation scores of rats given MS infusions of vehicle and hippocampal infusions of bicuculline $(\mathrm{V}-\mathrm{B})$ were not significantly different $(P>0.05)$. As expected, MS infusions of muscimol impaired memory. Specifically, compared with the percent alternation scores of $\mathrm{V}-\mathrm{V}$ rats, the percent alternation scores of rats given MS infusions of muscimol and hippocampal infusions of vehicle $(\mathrm{M}-\mathrm{V})$ were significantly decreased $(P<0.05)$. More importantly, hippocampal infusions of bicuculline prevented the alternation deficits produced by MS infusions of muscimol. Specifically, the percent alternation scores of rats given MS infusions of muscimol combined with hippocampal infusions of bicuculline (M-B) were not significantly different from those of $\mathrm{V}-\mathrm{V}$ rats $(P>0.05)$, but were significantly higher than those of $\mathrm{M}-\mathrm{V}$ rats $(P<0.05)$. Drug infusions into the MS $\left(F_{(1,42)}=0.542 ; P>0.05\right)$ and the hippocampus $\left(F_{(1,42)}=1.152 ; P>0.05\right)$ did not significantly affect the number of arms entered during SA, and the effect of drug infusions into both regions did not interact significantly $\left(\mathrm{F}_{(1,42)}=0.113 ; P>0.05\right.$ : Fig. $\left.6 \mathrm{~B}\right)$.

Drug infusions into the MS and hippocampus did not significantly affect trials to criterion during CMIA training $\left(\chi_{(3,36)}^{2}=6.45 ; P>0.05\right.$; Fig. 7A). Figure 7B illustrates that the pre-training drug infusions into the MS and hippocampus significantly affected subsequent CMIA retention performance $\left(\chi_{(3,36)}^{2}=11.74 ; P<0.01\right)$. Hippocampal infusions of bicuculline alone did not affect CMIA retention. That is, the retention latencies of $\mathrm{V}-\mathrm{B}$ rats were not significantly different from those of $\mathrm{V}-\mathrm{V}$ rats $\left(\mathrm{U}_{(1,16)}=26 ; P>0.05\right)$. Consistent with previous research, MS infusions of muscimol impaired CMIA retention. Specifically, $\mathrm{M}-\mathrm{V}$ rats had significantly shorter retention latencies than did $\mathrm{V}-\mathrm{V}$ rats $\left(\mathrm{U}_{(1,21)}=8 ; P<0.01\right)$. More importantly, hippocampal infusions of bicuculline attenuated the CMIA retention deficits produced by muscimol. The retention latencies of $\mathrm{M}-\mathrm{B}$ rats were not significantly shorter than those of $\mathrm{V}-\mathrm{V}$ rats $\left(\mathrm{U}_{(1,21)}=36.50\right.$; $P>0.05)$, but were significantly longer than those of $\mathrm{M}-\mathrm{V}$ rats $\left(\mathrm{U}_{(1,20)}=39.50 ; P>0.05\right)$.

\section{Discussion}

\section{Experiment 1}

The present findings show that MS infusions of scopolamine with muscimol, at doses that did not affect memory when infused alone, produced significant memory deficits when the two were infused together. This pattern of findings was observed in two different behavioral tasks. These findings are consistent with previous research showing that MS infusions of GABA agonists or ACh antagonists alone produce memory deficits (Chrobak et al. 1989; Givens and Olton 1990, 1995; Durkin 1992; Pang and

\section{A}

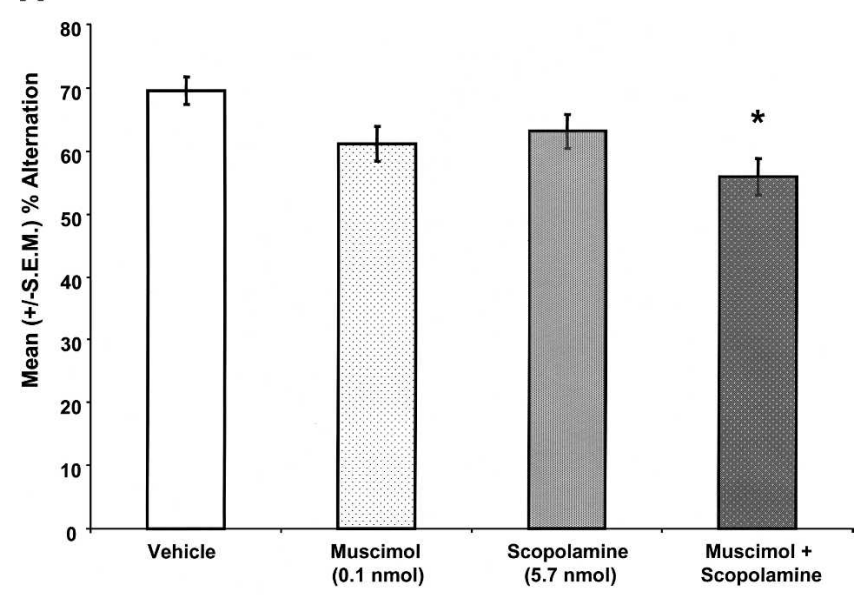

B

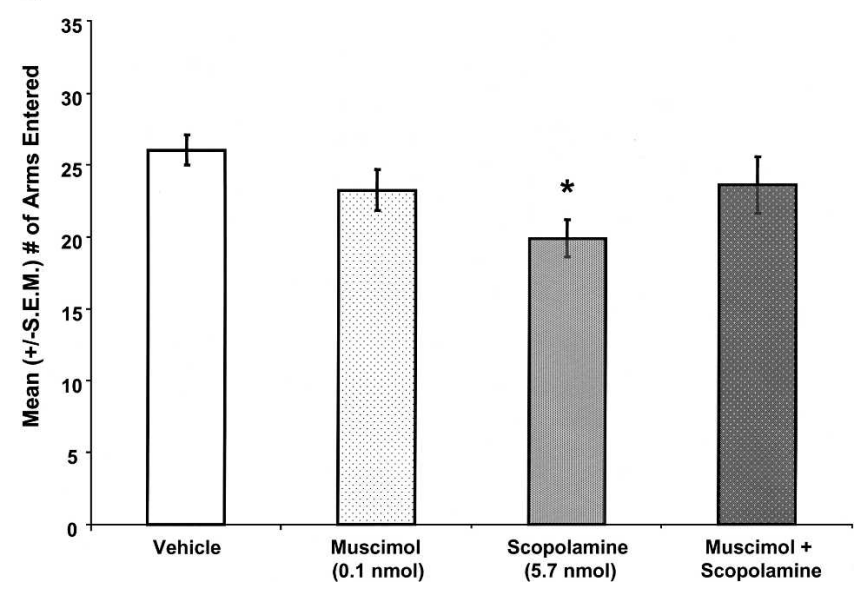

Figure 3. ( $A$ ) MS co-infusions of muscimol with scopolamine, at doses that had no effect on memory alone, significantly decreased mean ( \pm S.E.M.; $\mathrm{n}=14-16$ per group) percent alternation scores. $\left({ }^{*}\right) P<0.05$ vs. vehicle rats. $(B)$ MS infusions of scopolamine alone significantly decreased the mean ( \pm S.E.M.) number of arms rats entered in the maze. $\left(^{*}\right)$ $P<0.05$ vs. vehicle rats. 
A

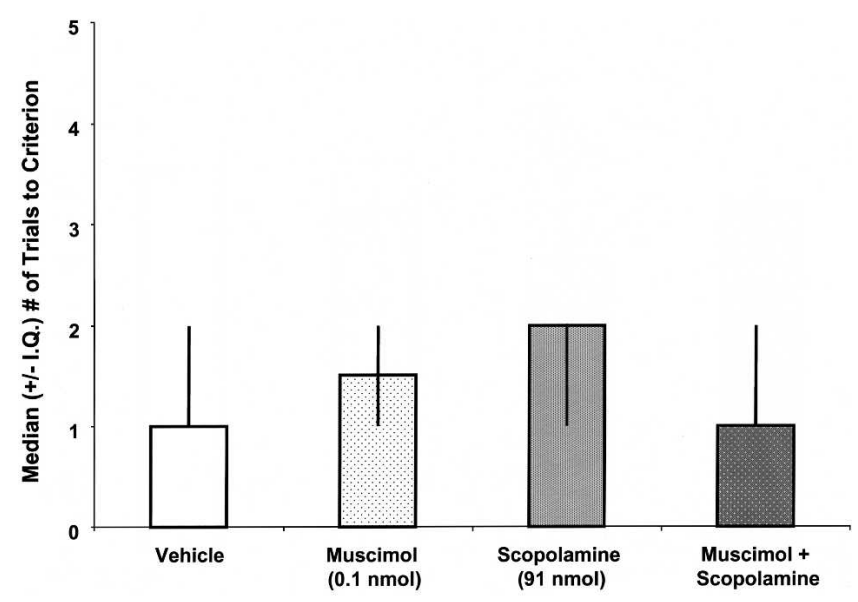

B

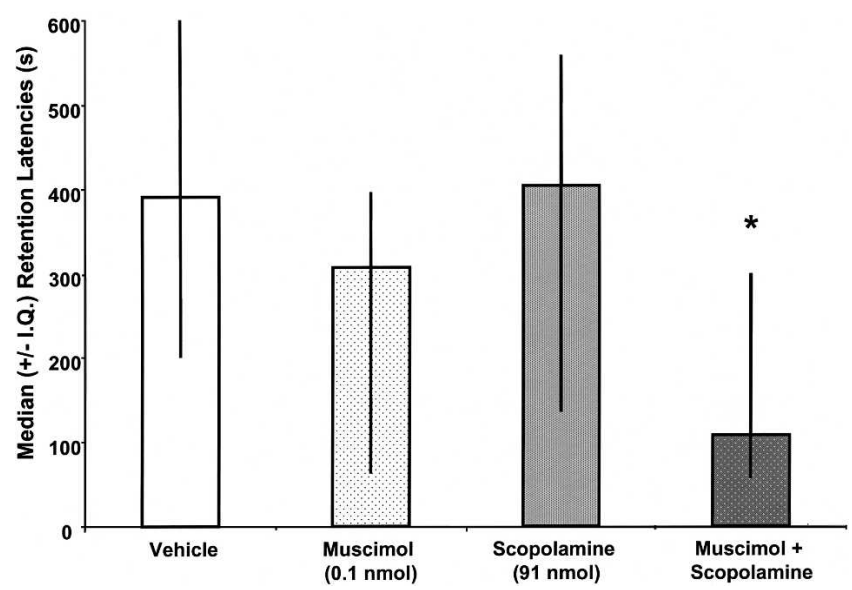

Figure 4. (A) There were no significant effects of MS infusions on median ( \pm I.Q.; $n=12-19$ per group) number of trials to criterion. ${ }^{*}$ ) $P>0.05$ vs. vehicle rats. (B) MS co-infusions of muscimol with scopolamine, at doses that had no effect on memory when infused alone, significantly decreased median ( \pm I.Q.) retention latencies. $\left(^{*}\right) P<0.05$ vs. vehicle rats.

Nocera 1999; Krebs and Parent 2005a,b). The present findings add to these previous results by showing that there is an interaction between the effects of scopolamine and muscimol in the MS. One interpretation of this finding is that scopolamine and muscimol each produced small subthreshold impairments via different mechanisms that summated when the two drugs were combined. Although the SA data are consistent with such an interpretation, the CMIA data suggest that the interaction between the effects of muscimol and scopolamine is synergistic rather than additive. That is, the CMIA data suggest that the two different drugs are acting at a common mechanism, and the SA data are also congruent with that construal. If one assumes that the same phenomenon is likely to occur in both tasks, then the most parsimonious interpretation of the findings of Experiment 1 is that the memory-impairing effects of MS GABA receptor activation involve GABAergic $\mathrm{SH}$ projection neurons. This is supported by electrophysiological evidence showing that septal infusions of scopolamine selectively influence GABAergic SH projection neurons (Alreja et al. 2000) and that GABA receptors are present on GABAergic SH projection neurons (Gao et al. 1995).

These data also showed that MS infusions of scopolamine decreased the activity of rats while they were performing on the memory task, which has been observed previously with systemic infusions of scopolamine (Masuoka et al. 2006). The scopolamine-induced decrease in the number of arms entered does not likely impact the interpretation of the SA data, because rats that were given MS infusions of scopolamine did not have impaired alternation scores. Moreover, MS co-infusions of scopolamine with muscimol affected alternation scores without affecting the mean number of arm entries.

\section{Experiment 2}

These results replicate the finding that MS infusions of the GABA receptor agonist muscimol impair performance in both SA and CMIA tasks (Chrobak et al. 1989; Durkin 1992; Parent and Gold 1997; Parent et al. 1997; Krebs and Parent 2005a,b). More importantly, the findings show that these memory deficits are reversed (SA) or attenuated (CMIA) by concurrent hippocampal infusions of the GABA antagonist bicuculline. Given that bicuculline would be expected to block input from GABAergic SH projection neurons, these findings suggest that GABAergic $\mathrm{SH}$ projection neurons are involved in the memory-impairing effects of MS GABA receptor activation.

\section{General discussion}

The present findings show that MS co-infusions of subeffective doses of muscimol with scopolamine impair SA and avoidance retention. MS administration of scopolamine selectively influences GABAergic SH projection neurons (Alreja et al. 2000). The present results also show that unilateral hippocampal infusions of bicuculline attenuate or prevent the memory-impairing effects of MS GABA receptor activation in these same memory tasks. Collectively, the combined findings provide converging evidence that GABAergic $\mathrm{SH}$ projection neurons are involved in the memory-impairing effects of septal GABA receptor activation.

These data are congruent with previous findings showing that unilateral hippocampal infusions are sufficient to reverse the memory-impairing effects of MS GABA receptor activation (Parent et al. 1997; Degroot and Parent 2000, 2001; Krebs and Parent $2005 a, b)$. These findings are also consistent with previous research suggesting that MS GABA receptor activation impairs memory via an influence on the hippocampus (Allen and Crawford 1984; Givens and Olton 1990; Durkin 1992; Walsh et al. 1993; Bland et al. 1996). It is important to note that the finding that hippocampal infusions of bicuculline prevent a memory deficit does not necessarily mean that the bicuculline was acting at the locus of the deficit. That is, it is possible that infusing a GABA antagonist into the hippocampus would have a positive effect on memory, regardless of whether or not GABA receptors are involved in the deficit. The most parsimonious interpretation that accounts for the data from both tasks and both sets of experiments, however, is that the impairing effects of septal GABA receptor activation involves GABAergic SH projection neurons. The finding that hippocampal infusions of bicuculline completely prevented muscimol-induced deficits in SA suggest further that GABAergic SH neurons may be necessary for the spatial working memory deficits produced by septal GABA receptor activation.

The present findings are also consistent with evidence showing that selective lesions of cholinergic SH projection neurons do not prevent the memory-impairing effects of MS GABA receptor activation (Pang and Nocera 1999). Rather, these lesions reduce the dose of muscimol needed to produce a memory deficit when infused into the MS (Pang and Nocera 1999), suggesting that MS GABA receptor activation impairs memory via a process that involves the GABA SH projection neurons. Combined with previ- 


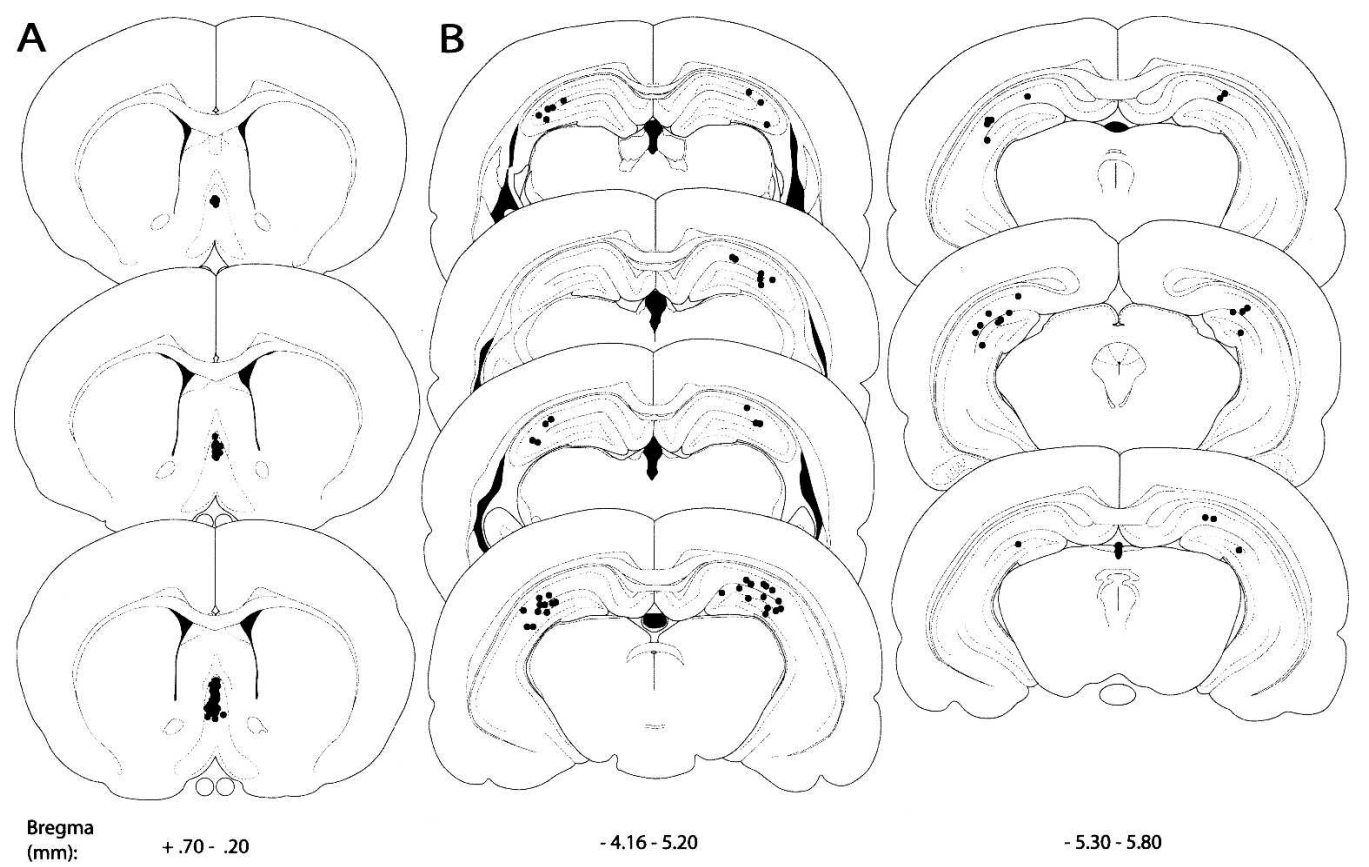

Figure 5. Schematic illustration of coronal sections of the rat brain showing the approximate location of MS $(A)$ and hippocampal (B) infusion sites in Experiment 2. Atlas plates were adapted from Paxinos and Watson (1998), with permission from Elsevier (01998.

ous electrophysiological findings (Alreja et al. 2000; Wu et al. 2000), the present findings suggest that MS manipulations that enhance or impair memory likely do so via a process that involves GABAergic SH projection neurons.

The interpretation that GABAergic SH projection neurons are involved in the memory-impairing effects of muscimol does not exclude the possibility that the impairing effects of septal GABA receptor activation also involve cholinergic SH projection neurons. MS infusions of muscimol decrease a variety of markers of hippocampal ACh (Allen and Crawford 1984; Walsh et al. 1993; Moor et al. 1998). More importantly, MS infusions of memory-impairing doses of muscimol decrease hippocampal extracellular ACh levels (Brioni et al. 1990; Durkin 1992; Degroot et al. 2003). Also, only those doses of muscimol that impair memory decrease hippocampal ACh function (Brioni et al. 1990). Furthermore, previous results suggest that hippocampal infusions of AChE inhibitors (Degroot and Parent 2000, 2001) or glucose (Parent et al. 1997; Krebs and Parent 2005a), which increase hippocampal ACh levels (Ragozzino et al. 1998), reverse memory deficits produced by MS GABA receptor activation. Thus, collectively, the evidence suggests that both projections are involved in the deficits produced by MS GABA receptor activation.

The hippocampal infusions of bicuculline may not have completely reversed the CMIA deficits because a larger dose of muscimol was infused in the CMIA task than in SA. The dose of bicuculline infused into the hippocampus was not increased in parallel with the higher dose of muscimol, because infusing a higher dose of bicuculline would increase the probability of seizure activity (Hu et al. 2005). It is not likely that the ability of bicuculline to reverse or attenuate the deficits involve seizure activity, because bicuculline had no effect on memory when infused alone.

To determine more definitively whether GABAergic SH projection neurons are required for the memory-impairing effects of MS GABA receptor activation, it would be ideal to selectively lesion GABAergic SH projection neurons and then administer muscimol into the MS. Recent findings show that MS adminis- tration of kainic acid may be one way to lesion GABAergic SH projection neurons. MS kainic acid lesions decrease the number of MS glutamate decarboxylase and parvalbumin immunoreactive cells (Pang et al. 2001; Dwyer et al. 2007). Parvalbumin immunoreactive cells in the MS are assumed to reflect GABA SH projection neurons (Freund and Antal 1988; Toth et al. 1993; Pang et al. 2001). These kainic-acid-induced lesions sometimes produce spatial working memory deficits (Dwyer et al. 2007) and sometimes do not (Pang et al. 2001). 192 IgG-induced lesions of cholinergic SH projection neurons produce only mild deficits (Pang et al. 2001; Dwyer et al. 2007; Fletcher et al. 2007). In contrast, combined lesions of the cholinergic and GABAergic SH projection neurons produce severe deficits (Pang et al. 2001). These findings suggest that the activity of either projection alone is sufficient but not necessary for memory. This may account for the finding that, although muscimol likely impairs memory by influencing both sets of projection neurons, bicuculline was able to reverse the memory deficit despite its apparent inability to influence the activity of cholinergic projection neurons. This would also imply that the fact that muscimol produced a memory deficit when combined with scopolamine would be attributable primarily to its ability to influence ACh projection neurons. It is important to note that these interpretations are based on the findings of lesion studies, which are always limited by the possibility that the lesions are incomplete and can induce compensatory changes. For example, recent evidence suggests that 192 IgG-induced lesions of cholinergic SH projection neurons do not completely deplete hippocampal extracellular ACh levels; moreover, there is an up-regulation of the residual ACh functioning (Chang and Gold 2004).

The motivational, temporal, and cognitive differences between the SA and CMIA tasks support the hypothesis that the drug infusions influenced memory rather than some other process such as attention or motivation that could influence performance in the memory tasks. The finding that MS GABA receptor activation impairs memory in both SA and CMIA indicates that the SH system is involved in several mnemonic processes. Specifically, the findings from the SA task suggest that the SH system 
A

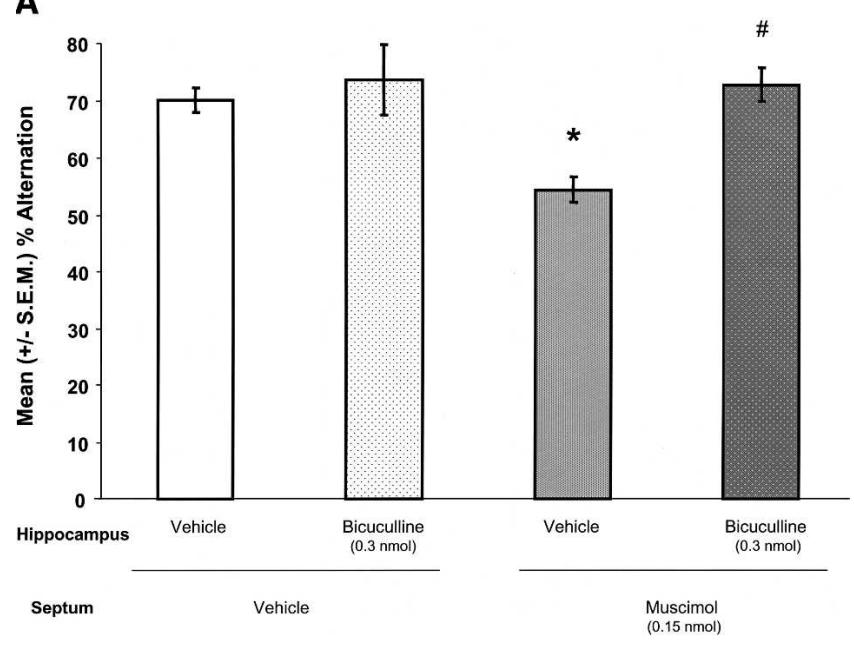

B

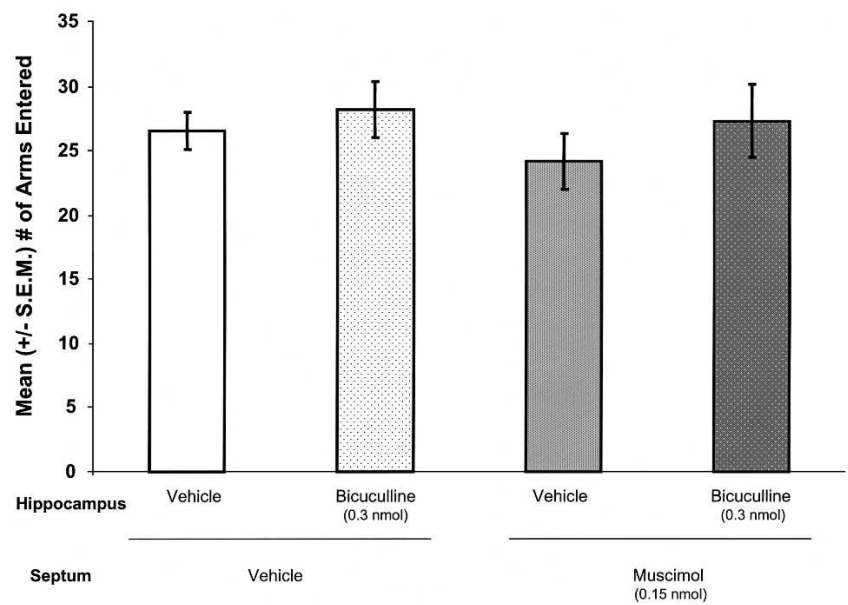

Figure 6. ( $A$ ) MS infusions of muscimol decreased mean ( \pm S.E.M.; $\mathrm{n}=9-12$ per group) percent alternation scores. $\left(^{*}\right) P<0.05$ vs. V-V. Hippocampal infusions of bicuculline, at a dose that did not affect percent alternation scores when infused alone, reversed the deficits produced by muscimol (\#) $P<0.05$ vs. $M-V, P>0.05$ vs. $V-V$. (B) There were no significant effects of any of the manipulations on the mean ( \pm S.E.M.) number of arm entries.

is involved in on-line spatial associations, and the findings from the CMIA task reveal that the SH system also affects emotional and long-term memory. The fact that the pre-training infusions of muscimol did not affect acquisition in the CMIA task suggests further that MS GABA receptor activation influences consolidation of newly formed emotional memories.

The present findings also show that higher doses of MS infusions of muscimol are needed to impair avoidance retention than SA. This is consistent with previous evidence investigating the effects of MS infusions of muscimol on different behavioral measures of memory (Brioni et al. 1990; Nagahara and McGaugh 1992; Parent and Gold 1997; Parent et al. 1997; Krebs and Parent 2005a,b). Collectively, this research shows that higher doses of muscimol are needed in the MS to impair shock avoidance than are needed to produce deficits in a rewarded alternation, SA, or spatial water maze task. It is not clear why higher doses are needed to impair shock avoidance. It is possible that the higher doses of muscimol may influence avoidance via non-GABAergic mechanisms or through diffusion to other brain regions. The finding that lower doses of muscimol are effective in impairing avoidance retention when they are co-infused with glucose, pyruvate (Parent and Gold 1997; Parent et al. 1997; Shah and Parent 2003, 2004), or scopolamine (present findings) suggests that this possibility is unlikely. The task-dependent effects of muscimol do not appear to be related to the stress level of the task, because both water maze and shock avoidance produce stress responses (De Boer et al. 1990; Mabry et al. 1995; Van der Borght et al. 2005). The differences also do not appear to be related to the presence of shock, because MS infusions of low doses of muscimol impair shock avoidance in a shock-probe burying test (Degroot and Treit 2003). Thus, these findings suggest that more MS GABA receptors or more prolonged MS receptor activation is required to impair avoidance memory that other types of memory. Additional research is needed to determine the factors that contribute to these task-dependent, dose-response differences.

In summary, the effects of MS co-infusions of scopolamine with muscimol combined to impair memory. Furthermore, intrahippocampal infusions of a GABA antagonist prevent the

A

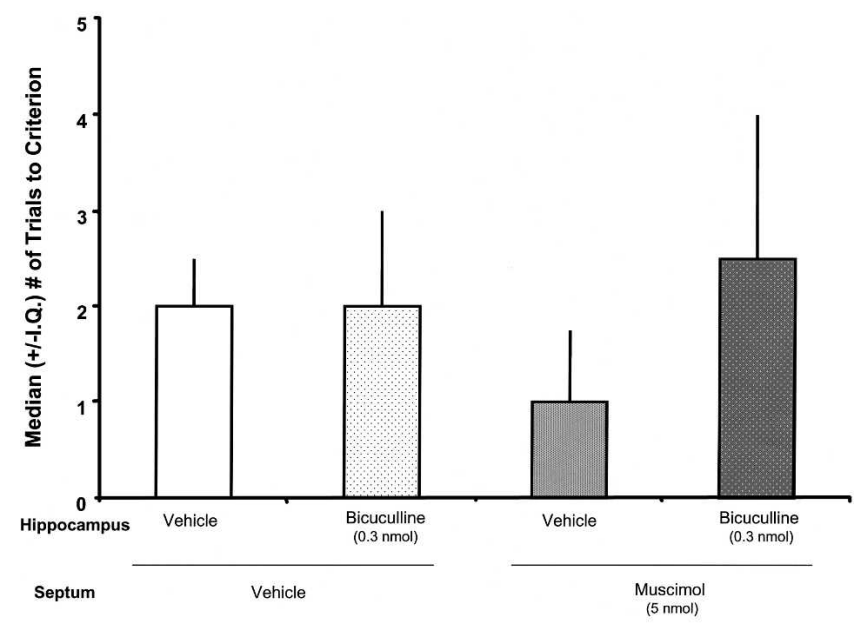

B

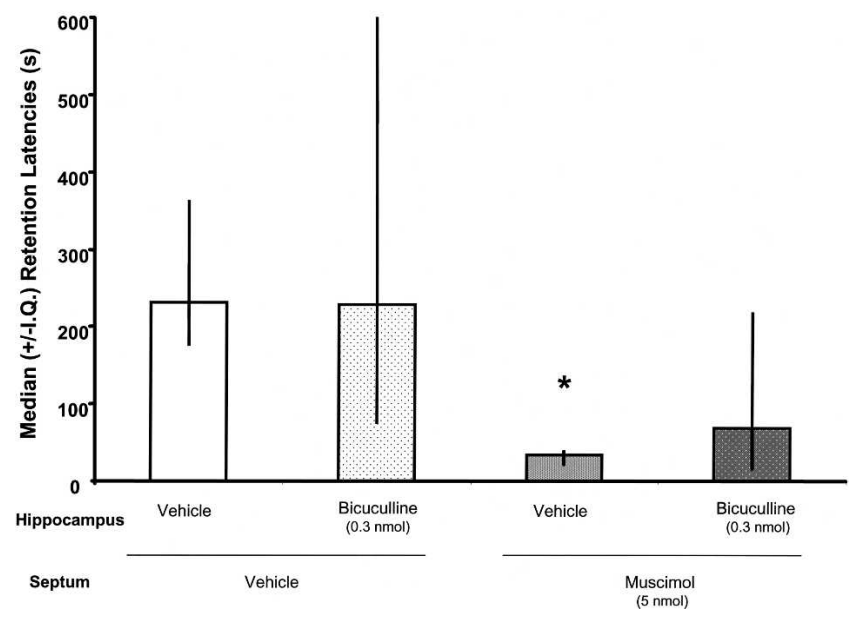

Figure 7. (A) There were no significant effects of any of the manipulations on the median ( \pm I.Q.; $\mathrm{n}=5-11$ per group) trials to criterion during CMIA training $(P>0.05$ vs. $V-V)$. (B) Pre-training MS infusions of muscimol decreased the median ( \pm I.Q.) retention latencies tested $48 \mathrm{~h}$ later. $\left(^{*}\right) P<0.05$ vs. V-V. Hippocampal infusions of bicuculline, at a dose that did not affect retention latencies when infused alone, attenuated the deficits produced by muscimol $(P>0.05$ vs. $\mathrm{M}-\mathrm{V}$ or $\mathrm{V}-\mathrm{V})$. 
memory-impairing effects of MS GABA receptor activation. These effects were observed in two different memory tasks. Collectively, these findings support the hypothesis that the effects of MS GABA receptor activation on memory are mediated through a process that requires GABAergic SH projection neurons. These studies are important because they constitute the first behavioral evidence indicating the GABAergic SH projection neurons are involved in both spatial working memory and emotional, longterm memory.

\section{Materials and Methods}

\section{Experiment 1}

The goal of this experiment was to determine whether GABAergic $\mathrm{SH}$ projection neurons are involved in the memory-impairing effects of septal GABA receptor activation. MS infusions of the muscarinic receptor antagonist scopolamine impair memory (Givens and Olton 1995; Pang and Nocera 1999; Elvander et al. 2004). Recent findings show that administration of scopolamine into the MS influences the activity of GABAergic, but not cholinergic, SH projection neurons (Alreja et al. 2000). When two drugs act via a common mechanism such as the GABAergic SH projection neurons to impair memory, then the effects of these two drugs should summate (Seeley and Moran 2002). We reasoned, therefore, that if memory-impairing effects of MS GABA receptor activation involve an influence on the GABAergic $\mathrm{SH}$ projection neurons, then co-infusions of muscimol and scopolamine, at doses that have no effect on memory alone, should produce memory deficits. Experiment 1 tested this prediction.

\section{Subjects}

Sixty-three male Sprague-Dawley-derived rats (Charles River) weighing 200-250 g upon arrival were used. The rats were housed individually in polycarbonate cages $(20 \times 40 \times 20 \mathrm{~cm})$ with corncob bedding on a 12-h light-dark cycle (lights on at 7:00 a.m.) in a temperature-controlled colony room $\left(70^{\circ} \mathrm{F}-74^{\circ} \mathrm{F}\right)$. Food and water were available ad libitum. The Georgia State University Institutional Animal Care and Use Committee (IACUC) approved all procedures involving rats.

\section{Surgery}

At least 1 wk after their arrival, the rats were given injections of atropine sulfate $(0.4 \mathrm{mg} / \mathrm{kg}$, ip, Baxter), anesthetized with sodium pentobarbital (Nembutal; $50 \mathrm{mg} / \mathrm{kg}$, ip, Abbott Laboratories), and then given an injection of penicillin (1500 units, im, Crystiben). The incision site was shaved with a \#50 electric clipper blade (Oster), and betadine solution was applied to the surgical area. The incision site was numbed with a $2 \%$ lidocaine/0.001\% epinephrine cocktail $(0.5-2.0 \mathrm{cc}$, sc, Abbott Labs). After the incision, the $2 \%$ lidocaine $/ 0.001 \%$ epinephrine solution was applied topically to the skull $(0.05-1.0 \mathrm{cc})$ to facilitate identification of lambda and bregma. Stereotaxic surgical procedures (David Kopf Instruments) were used to implant one 22-gauge stainless-steel guide cannula (Plastics One, Inc.) aimed at the MS $(0.5 \mathrm{~mm}$ anterior [AP] to bregma, $4.9 \mathrm{~mm}$ ventral to dura [DV]; Paxinos and Watson 1998). The cannulae were secured to the skull with three jeweler's screws and cranioplastic cement (DuraLay), and a dummy cannula (Plastics One, Inc.) was inserted to keep the cannulae free of debris. Immediately after surgery, the rats were given an injection of $0.9 \%$ sterile saline $(3.0 \mathrm{cc}, \mathrm{sc})$ and then wrapped with a paper towel and kept under a warm lamp until recovery from anesthesia. Two days following surgery, the patency of each cannula was checked, and betadine was applied to the surgical wound. If signs of infection were evident, the rats were anesthetized with isoflurane gas (5\%; Baxter) delivered in $1000 \mathrm{~mL} /$ minute of oxygen and given an additional injection of penicillin (1500 units im, Crystiben).

\section{Drug preparation and drug infusions}

Two days prior to behavioral testing, the experimenter handled each rat for 2 min. Before and after all handling and behavioral testing, the rats were allowed a minimum of $30 \mathrm{~min}$ to habituate to the laboratory environment. Behavioral testing occurred at least $1 \mathrm{wk}$ after surgery and was conducted between 7:00 a.m and 7:00 p.m. Drug treatments were counterbalanced over the course of the day. Fifteen minutes prior to assessing spontaneous alternation (SA) or training in continuous multiple trial inhibitory avoidance (CMIA), rats were given MS infusions of vehicle (phosphate-buffered saline [PBS]; $0.5 \mu \mathrm{L}, 0.5 \mu \mathrm{L} / \mathrm{min})$, muscimol $(0.10$ nmol: SA or $1 \mathrm{nmol}$ : CMIA), scopolamine (5.7 nmol: SA or 91 nmol: CMIA), or muscimol combined with scopolamine in the same solution. The drugs that were combined in the same solution were prepared at double the desired concentration and then combined, reducing the concentration of each by half. The doses of muscimol and scopolamine were selected based on our pilot data showing that these were the maximum doses that did not significantly impair memory when infused alone (D.L. KrebsKraft and M.B. Parent, unpubl.). The drugs were infused through a 28-gauge injection needle that extended $1.0 \mathrm{~mm}$ beyond the guide cannulae. The needle was connected to a $25-\mu \mathrm{L}$ Hamilton syringe by polyethylene tubing (PE-50), and the infusions were delivered using an infusion pump (Harvard Apparatus 11). Following the completion of both injections, the needle was left in place for $1 \mathrm{~min}$ to facilitate drug diffusion.

\section{Spontaneous alternation (SA)}

SA is assumed to be a hippocampal-dependent measure of spatial working memory (Stevens and Cowey 1973; Richman et al. 1987; Dember 1989; Deacon et al. 2002; Lalonde 2002). The underlying assumption is that in order to alternate successfully between locations, the rat must remember its visits to previous places. This assumption is supported in part by the finding that SA is impaired by removing directional cues or by increasing the interval between arm choices (Richman et al. 1987; Dember 1989; Lalonde 2002). Fifteen minutes after the drug injections, SA performance was assessed by placing each rat in a Y-maze composed of three equally spaced arms $\left(60^{\circ} ; 60 \mathrm{~cm}\right.$ long $\times 17.5 \mathrm{~cm}$ high $)$. The floor of each arm was composed of stainless steel $(3.5 \mathrm{~cm}$ wide), and the top (14 cm wide) was covered with a colorless, transparent plexiglass lid. All rats were placed in the same starting arm of the Y-maze and allowed to explore the maze for $8 \mathrm{~min}$. The experimenter, who was blind to drug treatment, recorded the sequence and number of arms the rats entered during the 8 min period. The maze was cleaned with $70 \%$ ethanol after each rat. The number of arms each rat entered was used as a measure of activity. A percent alternation score was computed for all rats that entered at least 10 arms. An alternation was defined as entering three different arms consecutively. The percent alternation score was computed by dividing the number of alternations each rat made by the number of arms entered minus two (i.e., the number of alternations possible) and then multiplying that resulting quotient by 100 .

\section{Continuous multiple trial inhibitory avoidance (CMIA)}

A minimum of $3 \mathrm{~d}$ after performing in the SA task, rats were trained on the shock avoidance task. The drug infusions were counterbalanced across the two behavioral tasks. The avoidance apparatus consisted of a trough-shaped alley $(91 \mathrm{~cm}$ long, $15 \mathrm{~cm}$ high, $20 \mathrm{~cm}$ wide at the top, and $6.4 \mathrm{~cm}$ wide at the bottom) that was divided into a lighted (31 cm long) and a dark (60 cm long) compartment by a retractable guillotine door. The dark compartment had a metal floor through which shock could be delivered. A 15-watt lamp was placed over the lighted compartment and was the only source of illumination in the room. The table underneath the avoidance apparatus was lined with bench paper and the apparatus was cleaned with $70 \%$ ethanol after each rat was trained or tested.

For the training, each rat was placed in the lighted compartment with its head facing away from the door. Once the rat turned around to face the door or after $12 \mathrm{sec}$ passed, the retractable door was opened and the rat was allowed to cross over to the dark (shock) compartment. After the rat crossed with all four paws, the rat was given a footshock $(1.2 \mathrm{~mA})$ until it returned to the lighted compartment (maximum $4 \mathrm{sec}$ ). This sequence con- 
stituted one training trial. Training continued until the rat remained in the lighted compartment for 100 consecutive sec or for a maximum of five trials. The rat was not removed from the avoidance apparatus between trials. The number of trials needed to reach the criterion was recorded and used as a measure of acquisition.

Retention of the training was tested $48 \mathrm{~h}( \pm 2 \mathrm{~h})$ later. Each rat was placed in the lighted compartment of the avoidance chamber with its head facing away from the closed door. After the rat turned to face the door or $12 \mathrm{sec}$ passed, the door was opened and the latency (sec) to cross over to the dark (shock) compartment was recorded and used as a measure of retention. Each rat was given a maximum of $600 \mathrm{sec}$ to enter the dark compartment, and foot shock was not delivered.

\section{Histology}

After behavioral testing, the rats were euthanized with an overdose of sodium pentobarbital (Sleepaway; $400 \mathrm{mg} / \mathrm{kg}$, ip) and perfused intracardially with $0.9 \%$ saline followed by $10 \%$ formalin. Their brains were stored in a $10 \%$ formalin solution for at least $2 \mathrm{~d}$ before sectioning. All brains were sectioned on a cryostat

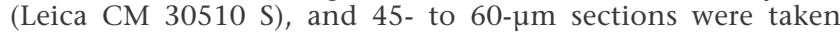
through the MS cannulae tracts. The brain sections were stained with thionin, and an unbiased observer determined the cannulae placement using a light microscope (Olympus BX41). Acceptable MS cannulae placement was defined as MS injection sites located within the MS, but not within the lateral septum or the ventral diagonal band of Broca. Moreover, the cannula must not have penetrated the fimbria.

\section{Statistical analysis}

The SA data were expressed as means and standard errors of the mean (S.E.M.) and analyzed using one-way analysis of variance (ANOVA) and Tukey post hoc tests where appropriate. The CMIA acquisition and retention latency data were not distributed normally due to the fact that several of the rats reached the maximum trials to criterion and/or $600 \mathrm{sec}$ retention latency cut-off. Consequently, these avoidance data were expressed as medians and interquartile ranges (I.Q.), and the nonparametric KruskalWallis and Mann-Whitney U-tests were used to detect differences between treatment groups. An alpha level of 0.05 was used as the criterion for statistical significance.

\section{Experiment 2}

The findings of Experiment 1 suggest that the memory-impairing effects of septal GABA receptor activation involve GABAergic $\mathrm{SH}$ projection neurons. The goal of Experiment 2 was to provide converging evidence to support that interpretation. Specifically, we determined whether hippocampal infusions of bicuculline, a $\mathrm{GABA}_{\mathrm{A}}$ receptor antagonist, which would be expected to block input from GABA neurons (Fig. 1B), would prevent memory deficits produced by concurrent MS infusions of muscimol.

The same procedures were used as in Experiment 1, with the following exceptions: Stereotaxic surgical procedures (David Kopf Instruments) were used to implant one 22-gauge stainlesssteel guide cannula (Plastics One, Inc.) aimed at the MS (0.5 mm AP to bregma, $4.9 \mathrm{~mm} \mathrm{DV}$ ) and one guide cannula aimed at the dorsal hippocampus ( $4.5 \mathrm{~mm} \mathrm{AP}, 1.6 \mathrm{~mm} \mathrm{DV}$, and $4.0 \mathrm{~mm}$ from the interaural line) (Paxinos and Watson 1998). The hemisphere in which the unilateral hippocampal cannulae were implanted was counterbalanced across rats. Fifteen minutes prior to behavioral testing, different groups of rats were given unilateral hippocampal infusions of vehicle $(1 \mu \mathrm{L}, 0.5 \mu \mathrm{L} / \mathrm{min}$; PBS, pH 7.4) or bicuculline methiodide ( $0.3 \mathrm{nmol}$; Sigma). The dose of bicuculline was selected based on pilot experiments showing that it was the highest dose that, when infused alone, did not affect memory or cause apparent seizure activity. One minute after the hippocampal infusion was initiated; the rats were given a MS injection of vehicle (PBS; $0.5 \mu \mathrm{L}, 0.5 \mu \mathrm{L} / \mathrm{min})$ or muscimol $(0.15 \mathrm{nmol}$ : SA or $5 \mathrm{nmol}$ : CMIA; Sigma). The doses of muscimol were selected based on findings showing that these doses decreased percent alternation scores and CMIA retention latencies without affect- ing activity measures or acquisition (Krebs and Parent 2005a,b). The drugs were infused through a 28-gauge injection needle that extended $1.2 \mathrm{~mm}$ (hippocampus) or $1.0 \mathrm{~mm}$ (MS) beyond the guide cannulae. The SA data were analyzed using a 2 (MS treatment) $\times 2$ (hippocampal treatment) univariate ANOVA and Tukey post hoc tests where appropriate.

\section{Acknowledgments}

This research was supported in part by grants from NINDSNIDDK-JDF (RO1NS41173-02), the STC Program of the National Science Foundation under Agreement No. IBN-9876754, and the Georgia State University Brains and Behavior Program. We thank Dana Donahue for her assistance in collecting the data.

\section{References}

Allen, C.N. and Crawford, I.L. 1984. GABAergic agents in the medial septal nucleus affect hippocampal theta rhythm and acetylcholine utilization. Brain Res. 322: 261-267.

Alreja, M., Wu, M., Liu, W., Atkins, J., Leranth, C., and Shanabrough, M. 2000. Muscarinic tone sustains impulse flow in the septohippocampal GABA but not cholinergic pathway: Implications for learning and memory. J. Neurosci. 20: 8103-8110.

Bilkey, D. and Goddard, G. 1985. Medial septal facilitation of hippocampal granule cell activity is mediated by inhibition of inhibitory interneurones. Brain Res. 361: 99-106.

Bland, B.H., Trepel, C., Oddie, S.D., and Kirk, I.J. 1996. Intraseptal microinfusion of muscimol: Effects on hippocampal formation theta field activity and phasic theta-ON cell discharges. Exp. Neurol. 138: $286-297$.

Brioni, J.D., Decker, M.W., Gamboa, L.P., Izquierdo, I., and McGaugh, J.L. 1990. Muscimol injections in the medial septum impair spatial learning. Brain Res. 522: 227-234.

Cahill, J. and Baxter, M. 2001. Cholinergic and noncholinergic septal neurons modulate strategy selection in spatial learning. Eur. J. Neurosci. 13: 1856-1864.

Chang, Q. and Gold, P.E. 2004. Impaired and spared cholinergic functions in the hippocampus after lesions of the medial septum/vertical limb of the diagonal band with 192 IgG-saporin. Hippocampus 14: 170-179.

Chrobak, J.J., Stackman, R.W., and Walsh, T.J. 1989. Intraseptal administration of muscimol produces dose-dependent memory impairments in the rat. Behav. Neural Biol. 52: 357-369.

Colom, L.V., Castaneda, M.T., Reyna, T., Hernandez, S., and Garrido-Sanabria, E. 2005. Characterization of medial septal glutamatergic neurons and their projection to the hippocampus. Synapse 58: 151-164.

De Boer, S.F., Van der Gugten, J., and Slangen, J.L. 1990. Brain benzodiazepine receptor-mediated effects on plasma catecholamine and corticosterone concentrations in rats. Brain Res. Bull. 24: $843-847$.

Deacon, R.M., Bannerman, D.M., Kirby, B.P., Croucher, A., and Rawlins, J.N. 2002. Effects of cytotoxic hippocampal lesions in mice on a cognitive test battery. Behav. Brain Res. 133: 57-68.

Degroot, A. and Parent, M. 2000. Increasing acetylcholine levels in the hippocampus or entorhinal cortex reverses the impairing effects of septal GABA receptor activation on spontaneous alternation. Learn. Mem. 7: 293-302.

Degroot, A. and Parent, M. 2001. Infusions of physostigmine into the hippocampus or the entorhinal cortex attenuate avoidance retention deficits produced by intra-septal infusions of the GABA agonist muscimol. Brain Res. 920: 10-18.

Degroot, A. and Treit, D. 2003. Septal GABAergic and hippocampal cholinergic systems interact in the modulation of anxiety. Neuroscience 117: 493-501.

Degroot, A., Kornecook, T., Quirion, R., DeBow, S., and Parent, M. 2003. Glucose increases hippocampal extracellular acetylcholine levles upon activation of septal GABA receptors. Learn. Mem. 979: 71-77.

Dember, W. 1989. Spontaneous alternation behavior. Springer-Verlag, New York.

Durkin, T. 1992. GABAergic mediation of indirect transsynaptic control over basal and spatial memory testing-induced activation of septo-hippocampal cholinergic activity in mice. Behav. Brain Res. 50: $155-165$.

Dwyer, T.A., Servatius, R.J., and Pang, K.C. 2007. Noncholinergic lesions of the medial septum impair sequential learning of different spatial locations. J. Neurosci. 27: 299-303.

Elvander, E., Schott, P., Sandin, J., Bjelke, B., Kehr, J., Yoshitake, T., and Ogren, S. 2004. Intraseptal muscarinic ligands and galanin: Influence on hippocampal acetylcholine and cognition. Neuroscience 
126: $541-557$

Farr, S., Uezu, K., Flood, J., and Morley, J. 1999. Septo-hippocampal drug interactions in post-trial memory processing. Brain Res. 847: 221-230.

Fletcher, B.R., Baxter, M.G., Guzowski, J.F., Shapiro, M.L., and Rapp, P.R. 2007. Selective cholinergic depletion of the hippocampus spares both behaviorally induced Arc transcription and spatial learning and memory. Hippocampus 17: 227-234.

Freund, T.F. and Antal, M. 1988. GABA-containing neurons in the septum control inhibitory interneurons in the hippocampus. Nature 336: $170-173$.

Frotscher, M. and Leranth, C. 1985. Cholinergic innervation of the rat hippocampus as revealed by choline acetyltransferase immunocytochemistry: A combined light and electron microscopic study. J. Comp. Neurol. 239: 237-246.

Gao, B., Hornung, J.P., and Fritschy, J.M. 1995. Identification of distinct GABAA-receptor subtypes in cholinergic and parvalbumin-positive neurons of the rat and marmoset medial septum-diagonal band complex. Neuroscience 65: 101-117.

Givens, B. and Olton, D. 1990. Cholinergic and GABAergic modulation of medial septal area: Effect on working memory. Behav. Neurosci. 104: 849-855.

Givens, B. and Olton, D. 1995. Bidirectional modulation of scopolamine-induced working memory impairments by muscarinic activation of the medial septal area. Neurobiol. Learn. Mem. 63: $269-276$

Gorman, L., Pang, K., Frink, K., Givens, B., and Olton, D. 1994. Acetylcholine release in the hippocampus: Effects of cholinergic and GABAergic compounds in the medial septal area. Neurosci. Lett. 166: $199-202$.

Hasselmo, M.E. 2005. What is the function of hippocampal theta rhythm? Linking behavioral data to phasic properties of field potential and unit recording data. Hippocampus 15: 936-949.

Hasselmo, M.E., Hay, J., Ilyn, M., and Gorchetchnikov, A. 2002. Neuromodulation, theta rhythm and rat spatial navigation. Neural Netw. 15: 689-707.

Hu, B., Karnup, S., Zhou, L., and Stelzer, A. 2005. Reversal of hippocampal LTP by spontaneous seizure-like activity: Role of group I mGluR and cell depolarization. J. Neurophysiol. 93: 316-336.

Izquierdo, I. and Medina, J.H. 1993. Role of the amygdala, hippocampus and entorhinal cortex in memory consolidation and expression. Braz. J. Med. Biol. Res. 26: 573-589.

Kohler, C., Chan-Palay, V., and Wu, J.Y. 1984. Septal neurons containing glutamic acid decarboxylase immunoreactivity project to the hippocampal region in the rat brain. Anat. Embryol. (Berl.) 169: 41-44.

Krebs, D.L. and Parent, M.B. 2005a. The enhancing effects of hippocampal infusions of glucose are not restricted to spatial working memory. Neurobiol. Learn. Mem. 83: 168-172.

Krebs, D.L. and Parent, M.B. 2005b. Hippocampal infusions of pyruvate reverse the memory-impairing effects of septal muscimol infusions. Eur. J. Pharmacol. 520: 91-99.

Krnjevic, K., Ropert, N., and Casullo, J. 1988. Septohippocampal disinhibition. Brain Res. 438: 182-192.

Lalonde, R. 2002. The neurological basis of spontaneous alternation. Neurosci. Biobehav. Rev. 26: 91-104.

Lewis, P.R., Shute, C.C., and Silver, A. 1967. Confirmation from choline acetylase analyses of a massive cholinergic innervation to the rat hippocampus. J. Physiol. 191: 215-224.

Mabry, T.R., Gold, P.E., and McCarty, R. 1995. Age-related changes in plasma catecholamine responses to chronic intermittent stress. Physiol. Behav. 58: 49-56.

Masuoka, T., Fujii, Y., and Kamei, C. 2006. Effect of scopolamine on the hippocampal theta rhythm during an eight-arm radial maze task in rats. Eur. J. Pharmacol. 539: 76-80.

Moor, E., Schirm, E., Jasco, J., and Westerink, B. 1998. Involvement of medial septal glutamate and GABAA receptors in behavior-induced acetylcholine release in the hippocampus: A dual probe microdialysis study. Brain Res. 789: 1-8.

Nagahara, A.H. and McGaugh, J.L. 1992. Muscimol infused into the medial septal area impairs long-term memory but not short-term memory in inhibitory avoidance, water maze place learning and rewarded alternation tasks. Brain Res. 591: 54-61.

O'Keefe, J. 1993. Hippocampus, theta, and spatial memory. Curr. Opin. Neurobiol. 3: 917-924.

Pang, K.C. and Nocera, R. 1999. Interactions between 192-IgG saporin and intraseptal cholinergic and GABAergic drugs: Role of cholinergic medial septal neurons in spatial working memory. Behav. Neurosci. 113: $265-275$.

Pang, K., Nocera, R., Secor, A., and Yoder, R. 2001. GABAergic septohippocampal neurons are not necessary for spatial memory. Hippocampus 11: 814-827.

Parent, M.B. and Gold, P.E. 1997. Intra-septal infusions of glucose potentiate inhibitory avoidance deficits when co-infused with the GABA agonist muscimol. Brain Res. 745: 317-320.

Parent, M., Laurey, P., Wilkniss, S., and Gold, P. 1997. Intraseptal infusions of muscimol impair spontaneous alternation performance: Infusions of glucose into the hippocampus, but not the medial septum, reverse the deficit. Neurobiol. Learn. Mem. 68: 75-85.

Paxinos, G. and Watson, C. 1998. The rat brain in stereotaxic coordinates. Academic Press, San Diego, CA.

Ragozzino, M.E., Pal, S.N., Unick, K., Stefani, M.R., and Gold, P.E. 1998. Modulation of hippocampal acetylcholine release and spontaneous alternation scores by intrahippocampal glucose injections. $J$. Neurosci. 18: 1595-1601.

Richman, C., Dember, W., and Kim, P. 1987. Spontaneous alternation behavior: A review. Curr. Psychol. Res. Rev. 5: 358-391.

Rye, D.B., Wainer, B.H., Mesulam, M.M., Mufson, E.J., and Saper, C.B. 1984. Cortical projections arising from the basal forebrain: A study of cholinergic and noncholinergic components employing combined retrograde tracing and immunohistochemical localization of choline acetyltransferase. Neuroscience 13: 627-643.

Seeley, R.J. and Moran, T.H. 2002. Principles for interpreting interactions among the multiple systems that influence food intake. Am. J. Physiol. Regul. Integr. Comp. Physiol. 283: R46-R53. doi: 10.1152/ajpregu.00021.2002.

Shah, A.A. and Parent, M.B. 2003. Septal infusions of glucose or pyruvate, but not fructose, produce avoidance deficits when co-infused with the GABA agonist muscimol. Neurobiol. Learn. Mem. 79: 243-251.

Shah, A.A. and Parent, M.B. 2004. Septal infusions of glucose or pyruvate with muscimol impair spontaneous alternation. Brain Res. 996: $246-250$

Smythe, J.W., Colom, L.V., and Bland, B.H. 1992. The extrinsic modulation of hippocampal theta depends on the coactivation of cholinergic and GABA-ergic medial septal inputs. Neurosci. Biobehav. Rev. 16: 289-308.

Sotty, F., Danik, M., Manseau, F., Laplante, F., Quirion, R., and Williams, S. 2003. Distinct electrophysiological properties of glutamatergic, cholinergic and GABAergic rat septohippocampal neurons: Novel implications for hippocampal rhythmicity. J. Physiol. 551: 927-943.

Stevens, R. and Cowey, A. 1973. Effects of dorsal and ventral hippocampal lesions on spontaneous alternation, learned alternation and probability learning in rats. Brain Res. 52: 203-224.

Teyler, T.J. 1987. Long-term potentiation and memory. Int. J. Neurol. 21-22: 163-171.

Toth, K., Borhegyi, Z., and Freund, T.F. 1993. Postsynaptic targets of GABAergic hippocampal neurons in the medial septum-diagonal band of broca complex. J. Neurosci. 13: 3712-3724.

Toth, K., Freund, T.F., and Miles, R. 1997. Disinhibition of rat hippocampal pyramidal cells by GABAergic afferents from the septum. J. Physiol. 500: 463-474.

Van der Borght, K., Meerlo, P., Luiten, P.G., Eggen, B.J., and Van der Zee, E.A. 2005. Effects of active shock avoidance learning on hippocampal neurogenesis and plasma levels of corticosterone. Behav. Brain Res. 157: 23-30.

Van der Zee, E.A. and Luiten, P.G. 1994. Cholinergic and GABAergic neurons in the rat medial septum express muscarinic acetylcholine receptors. Brain Res. 652: 263-272.

Vertes, R.P. and Kocsis, B. 1997. Brainstem-diencephalo-septohippocampal systems controlling the theta rhythm of the hippocampus. Neuroscience 81: 893-926.

Walsh, T.J., Stackman, R.W., Emerich, D.F., and Taylor, L.A. 1993. Intraseptal injection of GABA and benzodiazepine receptor ligands alters high-affinity choline transport in the hippocampus. Brain Res. Bull. 31: 267-271.

Wan, R.Q., Givens, B.S., and Olton, D.S. 1995. Opioid modulation of working memory: Intraseptal, but not intraamygdaloid, infusions of $\beta$-endorphin impair performance in spatial alternation. Neurobiol. Learn. Mem. 63: 74-86.

Wu, M., Shanabrough, M., Leranth, C., and Alreja, M. 2000. Cholinergic excitation of septohippocampal GABA but not cholinergic neurons: Implications for learning and memory. J. Neurosci. 20: 3900-3908.

Wu, W.W., Oh, M.M., and Disterhoft, J.F. 2002. Age-related biophysical alterations of hippocampal pyramidal neurons: Implications for learning and memory. Ageing Res. Rev. 1: 181-207.

Received October 16, 2007; accepted in revised form October 26, 2007. 


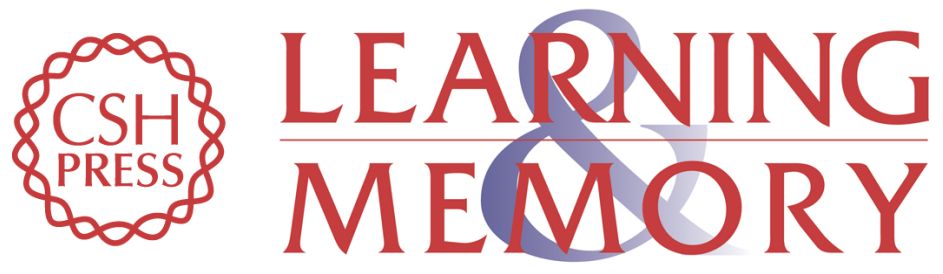

\section{The memory-impairing effects of septal GABA receptor activation involve GABAergic septo-hippocampal projection neurons}

Desiree L. Krebs-Kraft, Marina G. Wheeler and Marise B. Parent

Learn. Mem. 2007, 14:

Access the most recent version at doi:10.1101//m.809407

References This article cites 64 articles, 6 of which can be accessed free at:

http://learnmem.cshlp.org/content/14/12/833.full.html\#ref-list-1

License

Email Alerting Receive free email alerts when new articles cite this article - sign up in the box at the Service top right corner of the article or click here. 